\title{
Rapidly progressive post-transplant lymphoproliferative disease following withdrawal of sirolimus
}

\author{
M Mendelson, Z Barday, R Eastman, D Le Feuvre, S Candy, H Wu, C Swanepoel \\ Division of Infectious Diseases and HIV Medicine, Department of Medicine, Groote Schuur Hospital, Cape Town \\ M Mendelson, BSc, MB BS, PhD, FRCP (UK), DTM\&H
}

Division of Nephrology and Hypertension, Department of Medicine, Groote Schuur Hospital

Z Barday, MB ChB, FCP (SA), Cert Nephrol (SA)

C Swanepoel, MB ChB, MRCP (UK), FRCP (Edin)

Division of Neurology, Department of Medicine, Groote Schuur Hospital

R Eastman, MB ChB, FRCP (UK)

Department of Neurosurgery, Groote Schuur Hospital

D Le Feuvre, MB ChB, FCS (SA), MSc Neurosurgery, MMed

Department of Radiology, Groote Schuur Hospital

S Candy, FCRad (Diag) SA

Division of Anatomical Pathology, National Health Laboratory Service, Cape Town

H-T Wu, FCPath

Corresponding author: M Mendelson (Marc.mendelson@uct.ac.za)

Sirolimus, a potent inhibitor of B- and T-cell activation, is a commonly used immunosuppressant after renal transplantation. Withdrawal of sirolimus from the immunosuppression regimen may reduce B-cell surveillance. We present a case of rapidly progressive central nervous system (CNS) polymorphic Epstein-Barr virus (EBV)-related post-transplant lymphoproliferative disorder following the withdrawal of sirolimus.

S Afr Med J 2012;102(12):924-928. DOI:10.7196/SAMJ.5500

Post-transplant lymphoproliferative disorder (PTLD) occurs in $1-10 \%$ of patients undergoing solid-organ transplantation and presents predominantly in the first year. It is a heterogeneous entity and encompasses indolent B- or T-cell lymphoproliferation to aggressive monomorphic lymphoma. Sirolimus is an inhibitor of the response to interleukin-2 and blocks T- and B-cell activation. The drug has been used to treat renal transplant-related PTLD, but cases of PTLD related to stopping sirolimus have not been reported. We present a case of rapidly progressive central nervous system (CNS) PTLD 11 years after transplantation, in which sirolimus had been stopped 1 month prior to symptom onset.

\section{Case report}

The patient was a 31-year-old man who had undergone cadaveric renal transplantation 9 years previously for end-stage renal failure of undetermined cause. He presented with ataxia and acute confusion. In the 3 months preceding presentation he had experienced unexplained, asymptomatic weight loss.

Clinical examination was unremarkable. Sirolimus had been stopped 1 month before the onset of symptoms, owing to an apparent functional iron deficiency in the absence of evident gastro-intestinal blood loss. The patient remained on mycophenolate mofetil and prednisone.

On the morning of presentation, the patient's wife witnessed his confusion and unsteadiness. He was afebrile and normotensive, 

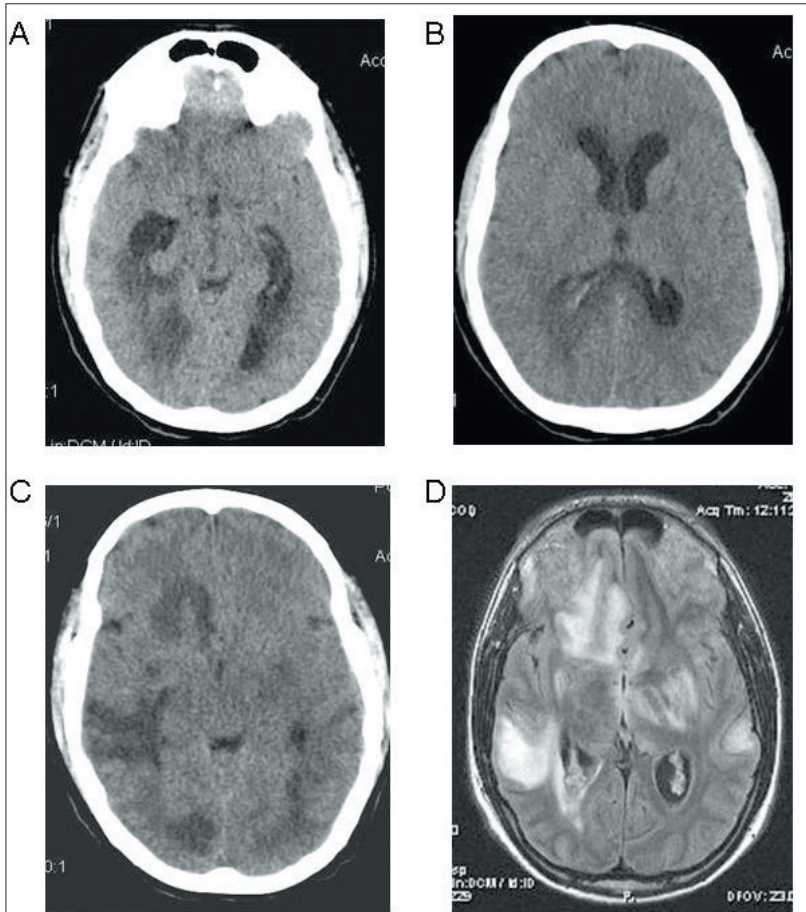

Fig. 1. (a) Non-enhanced CT (NECT) scan day 1, showing peri-trigonal white matter oedema; and early hydrocephalus. (b) NECT day 1, showing subtle prominence of the choroid plexus in the right trigone. (c) NECT day 9, depicting increased cerebral swelling with attenuation of the ventricles and bilateral, patchy, asymmetrical white matter at low densities. (d) FLAIR $M R I$ day 9, confirming patchy bilateral, predominantly white matter signal abnormalities.

with a normal examination apart from stiffness at the extreme of neck flexion and mild gait ataxia. He was fully orientated, but verbal responses were slow. Chest radiograph was normal, but a noncontrast CT scan (Figs 1A and 1B) showed subtle prominence of the choroid plexus in the right trigone, peritrigonal white matter oedema and early hydrocephalus. Lumbar puncture revealed 24 lymphocyte cells $/ \mathrm{mm}^{3}$, protein of $1.83 \mathrm{~g} / \mathrm{l}$, glucose $2.0 \mathrm{mmol} / \mathrm{l}$ and negative Gram stain, auramine phenol stain for Mycobacterium tuberculosis, India Ink stain and cryptococcal latex antigen tests. Urea was $18.2 \mathrm{mmol} / \mathrm{l}$, creatinine of $285 \mu \mathrm{mol} / \mathrm{l}$ and electrolytes were normal. Total white blood cell count was $8.4 \times 10^{9} / 1$ with lymphopenia of $0.54 \times 10^{9} / 1$.

The patient was started on empiric meningitis treatment with ceftriaxone, and ampicillin to cover Listeria monocytogenes. No neurosurgical intervention was indicated at the time. Subsequent CSF PCR for herpes simplex virus, cytomegalovirus, varicella zoster virus and $M$. tuberculosis were negative, as were toxoplasma and syphilis serology, as well as bacterial culture.

The patient deteriorated on day 5 of treatment, with spiking fever $>38^{\circ} \mathrm{C}$. Empiric antituberculosis therapy was added in light of the very high prevalence of the disease in the community, combined with the patient's history of unexplained weight loss and his clinical presentation. Prednisone dose was increased to $1.5 \mathrm{mg} / \mathrm{kg}$ in lieu of rifampicin. On day 9 , he became increasingly confused and incoherent, with a reduced score on the Glasgow coma scale. A repeat CT scan (Fig. 1C) revealed increased cerebral swelling with attenuation of the ventricles, as well as bilateral, patchy, asymmetrical white matter with low densities.

FLAIR magnetic resonance images obtained the same day (Fig. 1D) confirmed patchy, bilateral, predominantly white matter signal abnormalities.

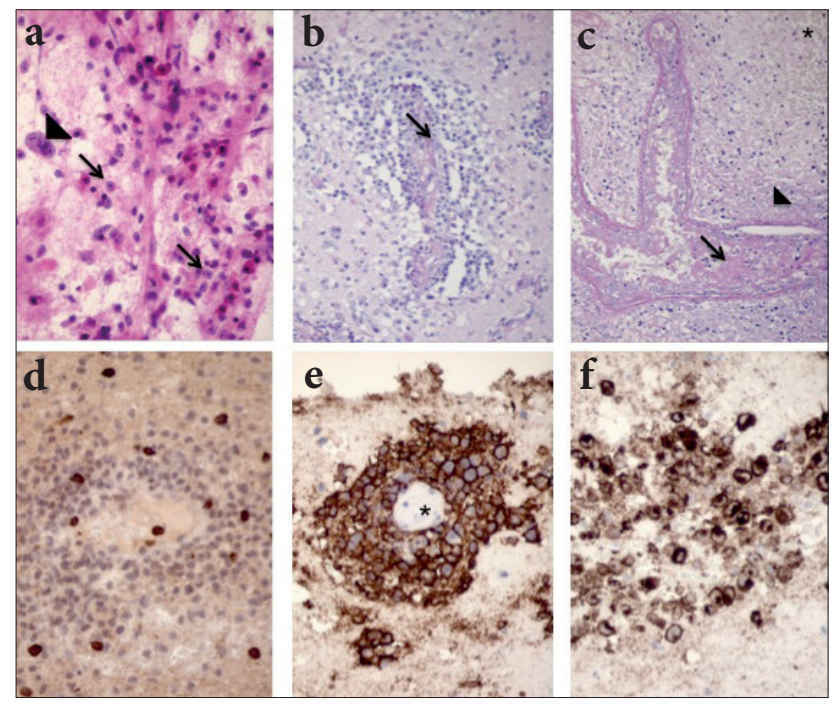

Fig. 2. Cytology, histology, and immunohistochemical findings. (a) Brain smear demonstrates predominantly small to medium-sized lymphocytes with prominent single nucleoli (arrows) and very occasional large lymphocytes (arrow head). Haematoxylin and eosin, 200x. (b) Paraffin section shows cortical brain tissue with an angiocentric infiltrate of small to mediumsized lymphocytes, which surrounds a vessel (arrow). Haematoxylin and eosin, 100x. (c) A thrombosed vessel with fibrin thrombi (arrow) is present, surrounded by infarcted brain tissue with coagulative necrosis (arrow head) and fresh haemorrhage (asterisk). Haematoxylin and eosin, 100x. (d) The CD3 immunostain highlights occasional small T-lymphocytes. CD3, 200x. (e) The CD20 immunostain marks the predominant population of B-lymphocytes, which cuffs a vessel (asterisk). CD30, 200x. (f) The EBV latent membrane antigen 1 immunostain labels the B-lymphocytes strongly, consistent with underlying EBV infection. EBV-LMP1, 200x.

Antimicrobial cover for nocardia, cryptococcus and herpes simplex infections was added. A further increase in intracranial pressure required the placement of an extra-ventricular drain and the patient was transferred to the ICU. A stereotactic brain biopsy was performed. The patient died 24 hours after admission to ICU from intractable raised intracranial pressure.

Full postmortem examination was not undertaken. However, brain biopsy (Fig. 2) showed cortical brain tissue with involvement by an angiocentric lymphoproliferative process. This was characterised by predominant perivascular infiltrate of polymorphous lymphocytes including small to large CD20positive B-lymphocytes with a few scattered small CD3positive T-lymphocytes. B-lymphocytes were mitotically active, predominantly medium-sized, and displayed mild to moderate nuclear atypia characterised by a prominent single nucleolus and irregular nuclear membranes. Also noted were occasional B-lymphocytes with markedly enlarged nuclei. The B-lymphocytes stained strongly for EBV latent membrane antigen 1. Adjacent brain tissue showed areas of coagulative necrosis, thrombosis, and fresh haemorrhage. Within the necrotic areas was neutrophil margination inside vessels with transmigration through the vessel walls, consistent with a vital response to an early brain infarction. There were no abscesses, granulomas or toxoplasma cysts. A periodic acid-Schiff (PAS) stain for fungi was negative.

\section{Discussion}

After skin cancer, PTLD is the second most common malignancy in transplanted adults. ${ }^{1}$ CNS manifestations occur in $22-30 \%$ 
of patients, and just over half of these affect only the brain. ${ }^{1,2}$ Presentation is often late, with mortality rates in excess of $60 \%{ }^{2}$

PTLD comprises a heterogeneous group of lymphoproliferative diseases, ranging from the early indolent disorders that resemble infectious mononucleosis, through to polymorphic PTLD and onto the more aggressive monormorphic B- and T-cell lymphomas, whether they are driven by EBV or not. ${ }^{3}$ Our patient's presentation was sub-acute, starting 1 month after the sirolimus-based regimen was switched to mycophenolate mofetil, when he started to lose weight. Thereafter he presented with rapid neurological deterioration. We hypothesise that acceleration of the lymphoproliferative process was induced by stopping sirolimus, which has potent effects on B-cells.

Sirolimus inhibits B- and T-cell activation by inhibiting intracellular signalling events downstream of the IL- 2 and other cytokine receptors. This arrests cell cycle progression at early $G_{1}$ phase and inhibits lymphocyte activation and proliferation., ${ }^{4,5}$ Interestingly, RAD (SDZ RAD), a derivative of sirolimus which shares the same mechanism of action, has been shown in both in vitro and in vivo murine models to have potent inhibitory activity on PTLD-like, human EBV-infected lymphoblastoid B-cell lines. ${ }^{6}$ RAD also increased the apoptotic rate of EBV-infected cells. Similarly, withdrawal of sirolimus in our patient might have lifted its inhibitory effect on EBV-infected B-cells that had already transformed into a polymorphic PTLD, allowing malignant progression of the disorder. Whether or not ciclosporin, a known risk factor for development of PTLD, played a part in the initial development of our patient's disease is also open to conjecture. Adding credence to our hypothesis, sirolimus has been used successfully in the treatment of EBV-induced B-cell PTLD occuring weeks after renal transplantation. ${ }^{7}$ Further, there is increasing recognition of the utility of newer inhibitors of the mammalian target of rapamycin (mTOR) such as everolimus, in the treatment of mantle cell lymphoma ${ }^{8}$ and other malignancies. ${ }^{9}$

Early recognition of PTLD allows earlier implementation of therapeutic strategies based on reducing immunosuppression..$^{10} \mathrm{CNS}$ PTLD may be the only manifestation of the malignancy. Therefore, in patients presenting with CNS symptoms post-transplant, PTLD should be considered in the differential diagnosis. If no other focus is found, it should prompt early investigation with neuro-imaging.

\section{References}

1. Boubenider S, Hiesse C, Goupy C, Kriaa R, Marchand S, Charpentier B. Incidence and consequences of post-transplantation lymphoproliferative disorders. J Nephrol 1997;10:136-145.

2. Penn I, Porat G. Central nervous system lymphomas in organ allograft recipients. Transplantation 1995;59:240-244.

3. Swerdlow SH, Webber SA, Chadburn A, Ferry JA. Post-transplant lymphoproliferative disorders (PTLD). In: WHO Classification of Tumours of Haematopoietic and Lymphoid Tissue (Swerdlow SH, Campo E, Harris NL, et al, eds.). Lyon: IARC, 2008:343-350.

4. Sehgal SN. Rapamune (RAPA, rapamycin, sirolimus): mechanism of action immunosuppressive effect results from blockade of signal transduction and inhibition of cell cycle progression. Clin Biochem 1998:31(5): 335-340.

5. Sakata A, Kuwahara K, Ohmura T, Inui S, Sakaguchi N. Involvement of rapamycin-sensitive pathway in CD40-mediated activation of murine B-cells in vitro. Immunol Lett 1999;68(2-3):301-309. [http://dx.doi.
Cakn org/10.1016/S0165-2478(99)00053-X]

6. Majewski M, Korecka M, Kossev P, et al. The immunosuppressive macrolide RAD inhibits growth of human Epstein-Barr virus-transformed B-lymphocytes in vitro and in vivo: A potential approach to prevention and treatment of post-transplant lymphoproliferative disorders. PNAS 2000;97(8):4285-4290. [http://dx.doi.org/10.1073/pnas.080068597] 10.1073/pnas.080068597

7. Cullis B, D'Souza R, McCullagh P, et al. Sirolimus-induced remission of post-transplant lymphoproliferative disorder. Am J Kidney Dis 2006;47(5):e67-72. [http://dx.doi.org/10.1053/j.ajkd.2006.01.029]

8. Haritunians T, Mori A, O'Kelly J, Luong QT, Giles FJ, Koeffler HP. Antiproliferative activity of RAD001 (everolimus) as a single agent and combined with other agents in mantle cell lymphoma. Leukemia 2007;21(2):333-339. [http://dx.doi.org/10.1038/sj.leu.2404471]

9. Ciuffreda L, Di Sanza C, Incani UC, Milella M. The mTOR pathway: a new target in cancer therapy. Curr Cancer Drug Targets 2010;10(5):484-495. [http://dx.doi.org/10.2174/156800910791517172]

10. Parker A, Bowles K, Bradley JA, et al. Management of post-transplant lymphoproliferative disorder in adult solid organ transplant recipients - BCSH and BTS Guidelines. Br J Haematol 2010;149:693-705.

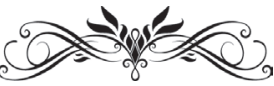

Three parts to this track: Forest, river, ancient shale Staircase of reflection.

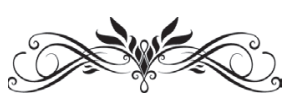

Wild mountain orchid Imperious, glowing red, For a fleeting week. 